\title{
BMJ Open Change to costs and lengths of stay in the emergency department and the Brisbane protocol: an observational study
}

Qinglu Cheng, ${ }^{1}$ Jaimi H Greenslade, ${ }^{1,2,3}$ William A Parsonage, ${ }^{1,2,3}$

Adrian G Barnett, ${ }^{1}$ Katharina Merollini, ${ }^{1}$ Nicholas Graves, ${ }^{1}$ W Frank Peacock, ${ }^{4}$ Louise Cullen ${ }^{1,2,3}$

To cite: Cheng $Q$,

Greenslade $\mathrm{JH}$

Parsonage WA, et al. Change to costs and lengths of stay in the emergency department and the Brisbane protocol: an observational study. BMJ Open 2016:6:e009746. doi:10.1136/bmjopen-2015009746

\section{- Prepublication history} and additional material is available. To view please visit the journal (http://dx.doi.org/ 10.1136/bmjopen-2015009746).

Received 19 August 2015 Revised 14 December 2015 Accepted 31 December 2015

CrossMark

For numbered affiliations see end of article.

Correspondence to Qinglu Cheng; qinglu.cheng@qut.edu.au

\section{ABSTRACT}

Objective: To compare health service cost and length of stay between a traditional and an accelerated diagnostic approach to assess acute coronary syndromes (ACS) among patients who presented to the emergency department (ED) of a large tertiary hospital in Australia.

Design, setting and participants: This historically controlled study analysed data collected from two independent patient cohorts presenting to the ED with potential ACS. The first cohort of 938 patients was recruited in 2008-2010, and these patients were assessed using the traditional diagnostic approach detailed in the national guideline. The second cohort of 921 patients was recruited in 2011-2013 and was assessed with the accelerated diagnostic approach named the Brisbane protocol. The Brisbane protocol applied early serial troponin testing for patients at 0 and $2 \mathrm{~h}$ after presentation to ED, in comparison with 0 and $6 \mathrm{~h}$ testing in traditional assessment process. The Brisbane protocol also defined a low-risk group of patients in whom no objective testing was performed. A decision tree model was used to compare the expected cost and length of stay in hospital between two approaches. Probabilistic sensitivity analysis was used to account for model uncertainty.

Results: Compared with the traditional diagnostic approach, the Brisbane protocol was associated with reduced expected cost of $\$ 1229$ (95\% Cl $-\$ 1266$ to $\$ 5122)$ and reduced expected length of stay of $26 \mathrm{~h}$ $(95 \% \mathrm{Cl}-14$ to $136 \mathrm{~h})$. The Brisbane protocol allowed physicians to discharge a higher proportion of low-risk and intermediate-risk patients from ED within $4 \mathrm{~h}(72 \%$ vs $51 \%$ ). Results from sensitivity analysis suggested the Brisbane protocol had a high chance of being costsaving and time-saving.

Conclusions: This study provides some evidence of cost savings from a decision to adopt the Brisbane protocol. Benefits would arise for the hospital and for patients and their families.

\section{INTRODUCTION}

Chest pain is a principal reason for adult emergency department (ED) visits ${ }^{1}$ with the

\section{Strengths and limitations of this study}

- This is the first study to report the changes to length of stay and cost from adopting an accelerated diagnostic approach for unspecified chest pain in Australian emergency departments.

- It was a large study that prospectively collected data on costs and outcomes.

- A decision tree model was developed to compare outcomes of the two approaches using realistic and clinically relevant patient pathways.

- Probabilistic sensitive analysis was used to account for uncertainties.

- This is an observational study and differences were found between the two cohorts that may confound differences due to the two approaches.

most common cause being acute coronary syndromes (ACS) including acute myocardial infarction (AMI) and unstable angina pectoris (UAP). Yet after thorough investigation most patients have non-cardiac conditions such as musculoskeletal pain or gastrointestinal causes for chest discomfort. In 20072008, 5.5 million people in the USA presented to EDs with chest pain and only $13 \%$ were diagnosed with ACS. ${ }^{2}$

Current management of patients with possible ACS in Australia arises from the National Heart Foundation and Cardiac Society of Australia and New Zealand Guidelines. $^{3} \quad$ Patients are stratified into low-risk, intermediate-risk and high-risk categories based on clinical features, ECG and troponin test results over a minimum of $6 \mathrm{~h}$ when using a sensitive troponin assay. Low-risk patients can be safely discharged. High-risk patients require admission to hospital and intensive management. Intermediate-risk patients form the largest group and further objective diagnostic testing to identify coronary artery disease (CAD) are required. The costs to health 
services and patient outcomes from these guidelines were described in a recent Australian study. ${ }^{4}$

The National Emergency Access Target (NEAT) was introduced in 2011 in Australia as part of the National Partnership Agreement on Improving Public Hospital Services. ${ }^{5}$ It requires $90 \%$ of all presentations to the ED to be discharged, admitted to hospital or transferred to another hospital for treatment within $4 \mathrm{~h}$. This target requires patients to be processed faster in the ED setting, and with the current guidelines requiring delayed troponin sampling, all patients with possible cardiac chest pain are steered towards admission to hospital.

Accelerated diagnostic protocols (ADPs) that risk stratify individuals within $2-3 \mathrm{~h}$ have recently been trialled. ${ }^{6-10}$ A large proportion of patients can be classified as low risk and rapidly referred for objective testing. ${ }^{6-8}{ }^{11}$ A study reporting on the implementation of the accelerated protocol found that average ED length of stay was reduced in the group of patients deemed low risk and health outcomes were maintained. ${ }^{9}$ Ongoing improvements in the assessment process of ED patients with chest pain have occurred and are in clinical use. ${ }^{12}$

A novel method for the assessment of ED patients with chest pain, the Brisbane protocol, was developed prior to the advent of NEAT in Australia. It was a clinician-led initiative in response to our improved clinical understanding of the impact of improvements in biomarker (troponin) assays and the unnecessary delays in testing during patient assessment. We believed that we could safely accelerate the assessment process and therefore designed the Brisbane protocol. This study compares the cost of managing patients for ACS who present to the ED under two competing configurations of health services: the traditional guidelines-based approach $^{3}$ and the Brisbane protocol. Detailed clinical outcomes of patients were not reported as this study focused on health economic outcomes of two diagnostic approaches.

\section{METHODS}

\section{Data collection}

This was an observational study that analysed data from two separate prospective patient cohorts presenting to the ED of a large tertiary hospital in Australia with possible ACS. The first patient cohort of 938 consenting patients was recruited in 2008-2010, and these patients were assessed using the traditional diagnostic approach detailed in the national guidelines. ${ }^{3}$ The main reason for recruiting the first cohort was to report on costs to health services and patient outcomes from applying the national guidelines. ${ }^{4}$ In this study, the first patient cohort was a baseline comparison group to assess the changes in the ED after the Brisbane protocol was designed and implemented. The second patient cohort $(\mathrm{n}=921)$ was recruited and assessed with the Brisbane protocol in 2011-2013. Process of care for patients managed by the traditional approach and the Brisbane protocol is shown in figure 1 .

Patients were recruited for both cohorts between 8:00 and 17:00 and were included if they were aged $\geq 18$ years, presented to the ED with at least $5 \mathrm{~min}$ of chest pain suggestive of ACS and were being investigated for ACS. In accordance with the American Heart Association case definitions, ${ }^{13}$ pain suggestive of ACS includes acute chest, epigastric, neck, jaw or arm pain, or discomfort or pressure without an apparent noncardiac source. Research staff identified all eligible patients using the ED admissions database and in collaboration with the treating clinicians. Patients were excluded if (1) there was a clear non-ACS cause for their symptoms, (2) they were unwilling or unable to provide informed consent such as a language barrier, (3) staff considered that recruitment was inappropriate, such as terminal illness, (4) they were transferred from another hospital, (5) they were pregnant, (6) they were recruited to the study within the previous 45 days or (7) they were unable or unwilling to be contacted after discharge. Perceived high risk was not an exclusion criterion. Consecutive eligible cases were included. The number of patients approached and the number of patients excluded for each reason in the first cohort have been published. ${ }^{4}$ In the second cohort, 1438 patients were approached. Excluded patients are as follows: 289 declined or were unable to consent, 72 were identified $>2 \mathrm{~h}$ after presentation, 39 were interhospital transfers, 17 were pregnant and 100 did not have cost data. Patients who were not eligible, who refused consent and who presented outside of recruitment periods were managed according to the historical guideline-based process of assessment.

Research nurses collected data on presentation date, admission date, discharge date, risk stratification and exercise stress test (EST) results. Total costs including the cost of the ED visit and any inpatient costs were extracted from a linked administrative database. Thirty days after initial attendance, research nurses conducted telephone follow-up and medical record review for the diagnosis of ACS. Information was obtained from the patient and from hospital databases about whether there had been any cardiac events or investigations, or contact with any healthcare providers, during the 30-day period. All follow-up information was verified through contact with the healthcare provider, and original copies of medical records and cardiac investigation results were obtained. Relevant investigations included EST, stress echocardiography, myocardial perfusion scanning, coronary CT angiography or coronary angiography. The 30-day clinical outcomes were adjudicated independently by at least one of two local cardiologists using predefined standardised reporting guidelines. ${ }^{14}$ Cardiologists had knowledge of all clinical information collected within a 30-day period. For both cohorts, this included all hospital medical records, public and private 
Traditional diagnostic approach

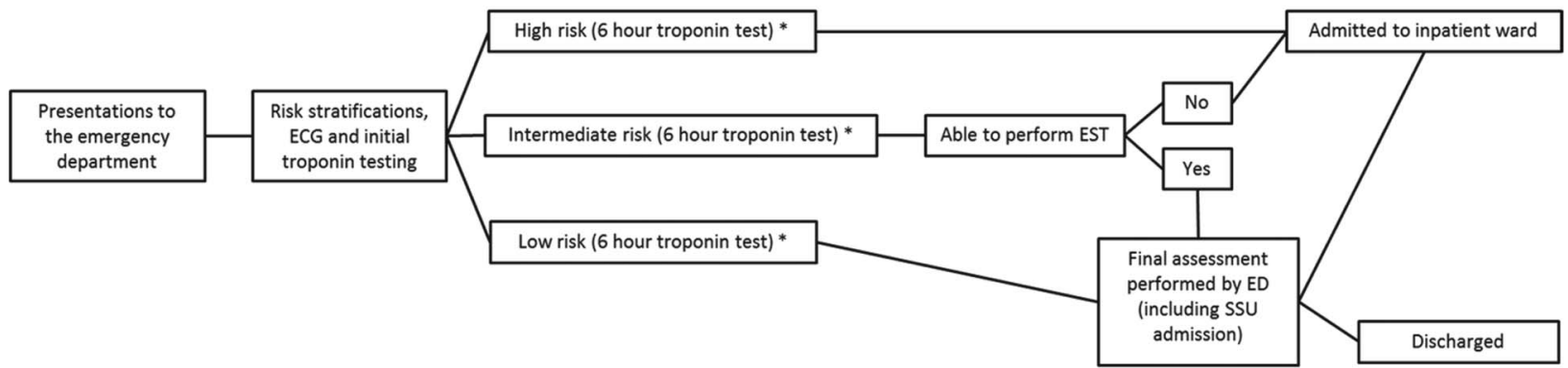

Brisbane protocol

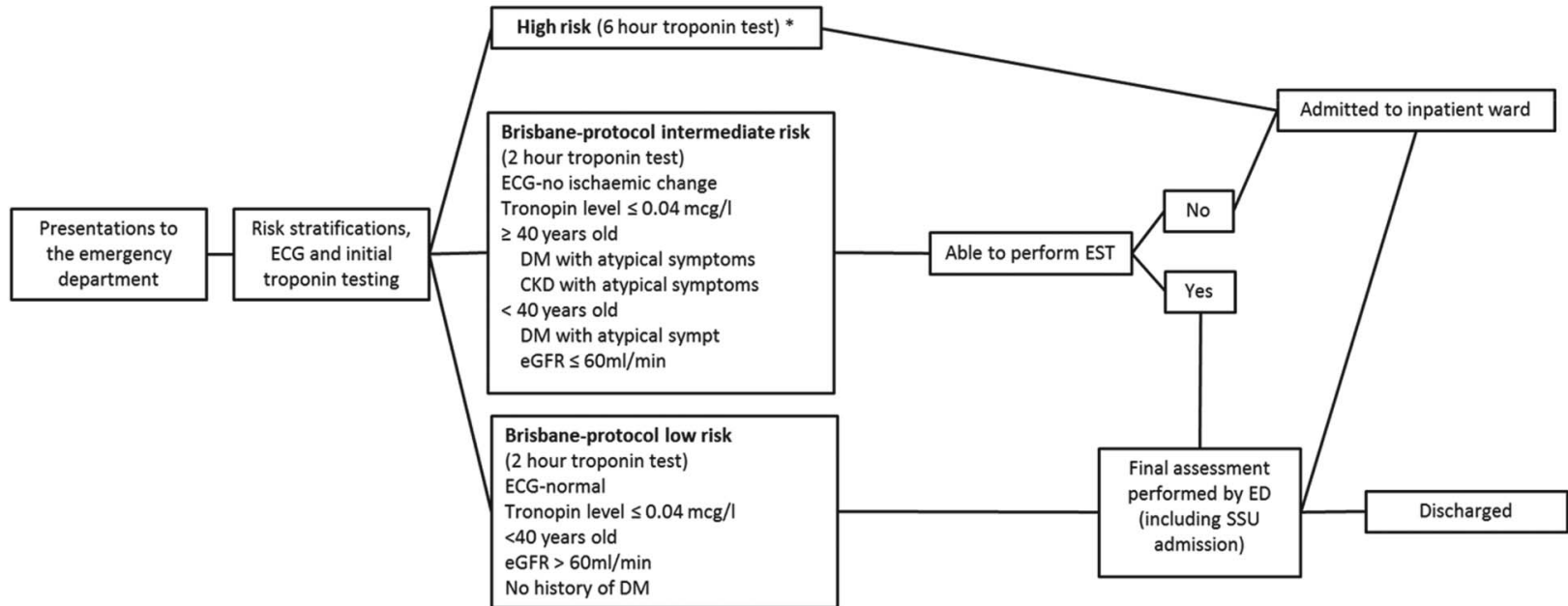

* Manage per National Heart Foundation and Cardiac Society of Australia and New Zealand Guidelines. ECG $=$ electrocardiography. EST $=$ exercise stress test. ED $=$ emergency department. $\mathrm{SSU}=$ short stay unit. $\mathrm{DM}=$ diabetes mellitus. $\mathrm{CKD}=$ chronic kidney disease. eGFR = estimated glomerular filtration rate

Figure 1 Process of care for patients with possible acute coronary syndromes under the traditional approach and the Brisbane protocol.

investigations, details provided by general practitioners and specialists seen within 30 days after discharge and by telephone contact with patients. In the first cohort, a second cardiologist conducted a blind review of all ACS cases and a random sample of $10 \%$ of non-ACS cases. In cases of disagreement, end points were agreed by consensus. This was achieved for all end points. For the second cohort, a single cardiologist completed end point adjudication as the second adjudication of the outcomes has not occurred at this point in time. The clinical outcomes will be fully reported once this second adjudication has occurred. Diagnosis of AMI and UAP was based on accepted international standards as described previously. ${ }^{15}$

\section{Decision tree model}

A decision tree model was developed to compare costs and health outcomes of the two approaches using realistic and clinically relevant patient pathways. The model enabled the change to costs and health outcomes to be clearly presented, and the uncertainties in the data to be included. The purpose of the model was to inform a decision between the Brisbane Protocol and the traditional approach. The traditional approach based on national guidelines ${ }^{3}$ is shown in figure 2. All non-high-risk patients were initially stratified into intermediate-risk and low-risk categories based on clinical features, ECG findings and troponin results obtained on presentation. Ongoing clinical assessment and repeat ECG and troponin testing were performed $6 \mathrm{~h}$ later. Low-risk patients were discharged and costs arising from the index presentation were included. After serial troponin and ECG testing $6 \mathrm{~h}$ after presentation were normal, patients in the intermediate-risk group were referred for EST; however, owing to clinical reasons, some intermediate-risk patients did not have this test. If the EST result was positive, patients were further stratified to high risk and admitted to an inpatient bed. If negative, patients were considered low risk and discharged home. Patients with an equivocal EST and who were discharged within $24 \mathrm{~h}$ were defined as low risk, and those discharged $>24 \mathrm{~h}$ were defined as high risk. Patients who did not have an EST were either directly admitted to an inpatient bed or discharged home after appropriate management in the ED and/or ED short stay unit. A small number of patients left against medical advice before treatment started. 


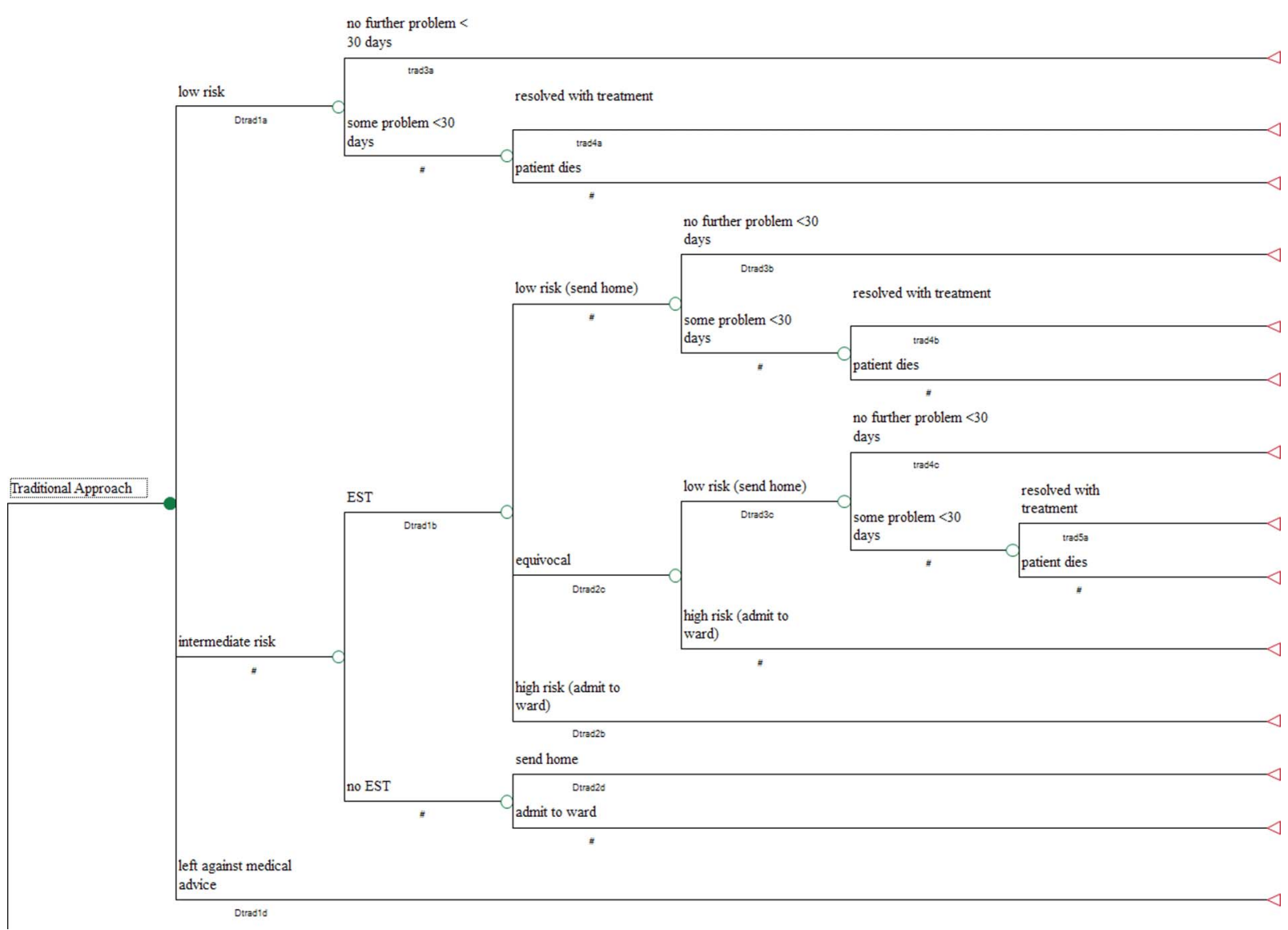

Figure 2 Traditional approach pathways.

A fundamental change in the new assessment process was the introduction of early serial troponin testing at 0 and $2 \mathrm{~h}$ after presentation for low-risk and intermediate-risk patients, in comparison with the traditional 0 and $6 \mathrm{~h}$ testing. The alternate Brisbane protocol is shown in figure 3. High-risk patients were initially identified and managed according to the traditional approach since the Brisbane protocol was designed for low-risk and intermediate-risk patients. All non-high-risk patients were then assessed using the Brisbane protocol. Those under 40 years of age without diabetes or renal impairment were defined as Brisbane protocollow risk, while the rest were classified as Brisbane protocol-intermediate risk. Patients in the Brisbane protocol-intermediate-risk group were referred for EST. As this was a pragmatic study design, some patients from the Brisbane protocol-low-risk group were also referred for EST based on individual patient characteristics. If the EST was positive, the patient was considered high risk and admitted to an inpatient bed. If negative, patients were discharged and any problems within 30 days were included. If equivocal and discharged within $24 \mathrm{~h}$, patients were defined as low risk. If they were admitted $>24 \mathrm{~h}$, they were categorised as high risk. Patients who were not referred for an EST were either admitted to an inpatient bed or discharged home after appropriate management in the ED/ED short stay unit.
Again, only a small number of patients left against medical advice.

The decision trees are designed to summarise expected costs and hospital length of stay under the traditional approach and the Brisbane protocol to give a system-level picture of the costs and benefits that would be useful to a high-level decisionmaker. If there are differences in the number of deaths, this is also shown quantitatively by the decision tree. Clinicians working in the ED validated the structure of the decision tree model prior to data analysis.

\section{Data analysis}

Age, gender, risk factors and medical history were compared across the two cohorts. The primary outcomes are health service cost and length of stay in hospital and were compared using the decision tree model. As the Brisbane protocol is for low-risk to intermediate-risk patients, all high-risk patients were managed according to the current National Heart Foundation and Cardiac Society of Australia and New Zealand Guidelines ${ }^{3}$ and were excluded from the analysis. The proportion of patients discharged from ED within $4 \mathrm{~h}$ was compared to show if the Brisbane protocol was associated with improved performance against the NEAT target.

This was a historically controlled study without random assignment; hence, there may have been 


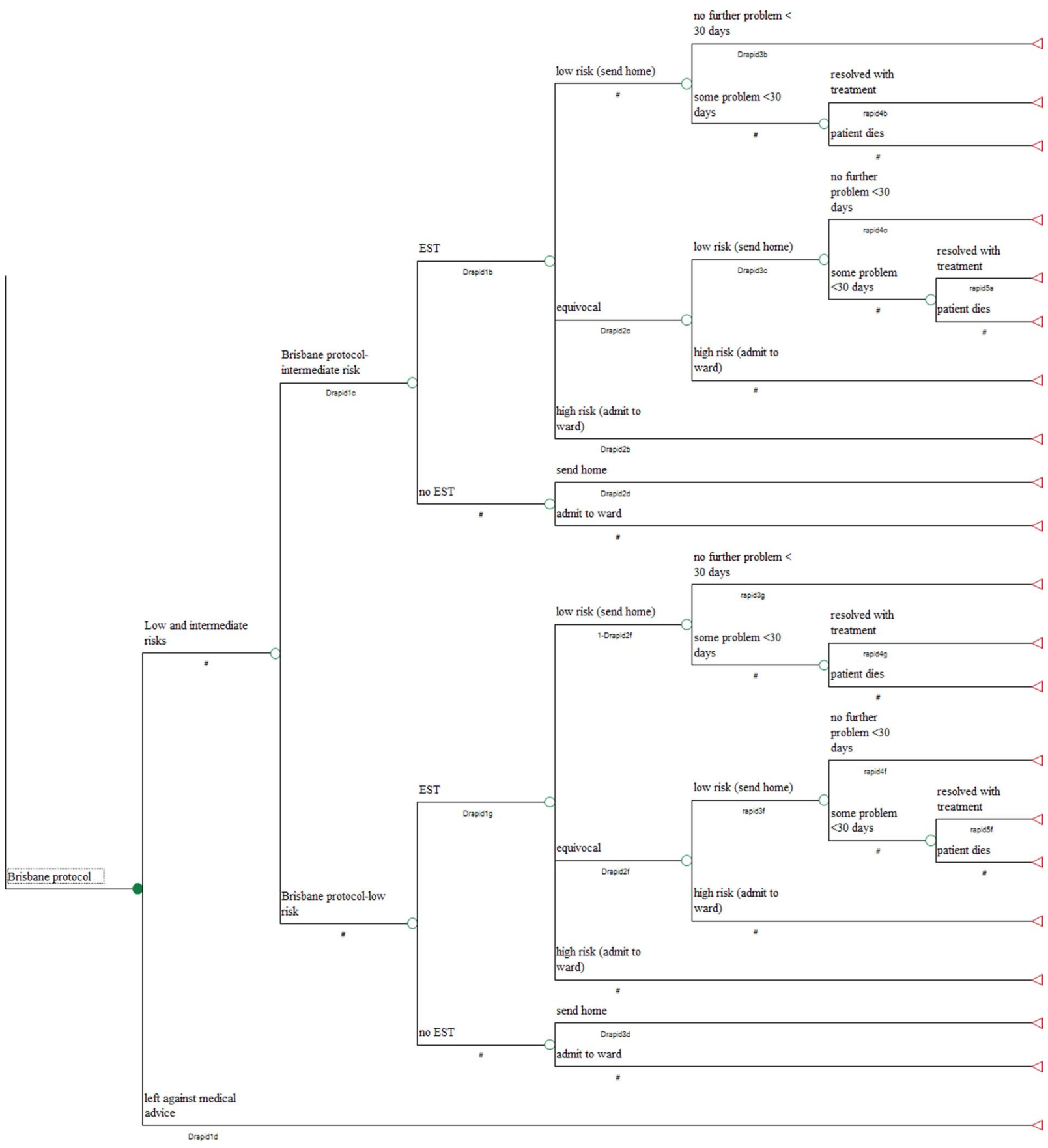

Figure 3 Brisbane protocol pathways.

differences between the two cohorts at baseline. We used multiple variable regression models to test if baseline characteristics were associated with risk stratification, cost, length of stay in hospital and proportion of patients discharged from ED within $4 \mathrm{~h}$ (in IBM SPSS Statistics 21). Results of regression analysis are provided in the online supplementary material. To test whether more patients risk stratified to low risk was due to baseline characteristics or the Brisbane protocol, we used binary logistic regression as the new stratification only works on low-risk and intermediate-risk patients. The results suggest that it was the Brisbane protocol that was mainly responsible for the change in risk stratification, so any difference in baseline characteristics should not have greatly impacted risk stratification. We also used linear regression to test if baseline characteristics had any impact on cost and length of stay for patients who moved through the same pathway in the decision tree model (eg, patients who were classed as intermediate risk, had EST and had negative EST outcome). The results suggested little impact from baseline characteristics on total costs and length of stay in hospital. This is probably because patients who moved through the same pathway in the decision tree were relatively homogeneous. Thus, baseline differences between patients had less potential to influence costs and length of stay. 
Therefore, we did not adjust decision tree model inputs by baseline characteristics.

Differences between two cohorts at baseline did influence the proportion of patients discharged from ED within $4 \mathrm{~h}$. To account for this, we used iterative poststratification to match the marginal distributions of the traditional approach cohort to the Brisbane protocol cohort. The variables matched were age (10-year bands), gender, prior myocardial infarction (MI), prior angina, prior $\mathrm{CAD}$, prior arrhythmia, prior congestive heart failure $(\mathrm{CHF})$, prior hypertension, prior dyslipidaemia and prior family CAD. We then calculated the per cent discharged within $4 \mathrm{~h}$ between the two cohorts using the post-stratification weights and compared this with an unweighted per cent. We used the 'rake' function in the 'survey' library in R. ${ }^{16}$

\section{Updating the decision tree with information}

The probabilities associated with the events at each circular chance node in the decision trees were derived from the two patient cohorts. The estimated probabilities were the risk of patients having low or intermediate risk, undergoing EST, having positive, negative or equivocal EST results, being admitted to inpatient ward or being discharged. Prior $\beta$ distributions that can only take values between 0 and 1 were used to model the probabilities and the uncertainty.

The costs incurred for the ED and inpatient wards were retrieved from each patient's hospital administration record that had been linked to the primary patient data. ED costs that include a fixed cost and an activitybased component were based on triage categories of clinical urgency. ${ }^{4}$ Inpatient costs were derived from procedure-related Australian Refined Diagnosis Related Group reimbursement codes used for activity-based funding. ${ }^{4}$ These costs were summed for each individual. For patients who moved through a common pathway in the decision tree, the median cost values were calculated to inform the cost outcome of that path. The costs of adverse events that might occur after discharge were not included. A prior $\gamma$ distribution was fitted to these data to capture the inherent skew in cost data. ${ }^{17}$ Costs from 2008 to 2012 were adjusted by an inflation rate of $3.4 \%$ per year to equal 2013 prices. ${ }^{18}$ Lengths of stay in hospital were derived from dates of presentation and discharge and were also fitted to $\gamma$ distributions.

Expected costs and lengths of stay are based on the summation of the pathway cost and hours in hospital weighted by the pathway probabilities. By comparing the expected cost and length of stay of the two competing diagnostic approaches, we defined the costs and time spent in ED when the Brisbane protocol was used.

A probabilistic sensitivity analysis was used to account for uncertainty in the information used in the model. Resampling was done 10000 times from the prior distributions using Monte Carlo simulation with cost and length of stay varying simultaneously. The probability of an approach being optimal was derived by counting the number of times out of 10000 the approach had lower costs or shorter length of hospital stay.

\section{RESULTS}

\section{Patient characteristics}

The baseline patient characteristics for both cohorts are shown in table 1. Patients in the traditional approach group were older and suffered more frequently from

\begin{tabular}{lccr} 
Table 1 Baseline characteristics by cohort & \multicolumn{1}{c}{ Brisbane } \\
Variable & $\begin{array}{l}\text { Traditional } \\
\text { approach }(\mathbf{n = 9 3 8 )}\end{array}$ & $50.8(12.9)$ & $\mathbf{p}$ Value \\
\hline Age, mean (SD) & $54.8(15.1)$ & $538(58.4)$ & $<0.01$ \\
Male sex, $\mathrm{n}(\%)$ & $573(61.1)$ & $306(33.2)$ & 0.24 \\
Risk factors $\mathrm{n}(\%)$ & $396(42.2)$ & $320(34.7)$ & $<0.01$ \\
Hypertension & $391(41.7)$ & $105(11.4)$ & $<0.01$ \\
Dyslipidaemia & $115(12.3)$ & $352(38.3)$ & 0.57 \\
Diabetes & $434(46.3)$ & $267(29.0)$ & $<0.01$ \\
Family history of CAD & $259(27.6)$ & & 0.51 \\
Current smoking & & $115(12.5)$ & $<0.01$ \\
Prior medical history $\mathrm{n}(\%)$ & $158(16.8)$ & $99(10.7)$ & $<0.01$ \\
Prior MI & $211(22.5)$ & $74(8.0)$ & 0.04 \\
Prior angina & $101(10.8)$ & $31(3.4)$ & $<0.01$ \\
Prior angioplasty & $58(6.2)$ & $11(1.2)$ & 0.16 \\
Prior CABG & $19(2.0)$ & $12(1.3)$ & $<0.01$ \\
Prior peripheral arterial disease & $43(4.6)$ & $49(5.3)$ & $<0.03$ \\
Prior CHF & $83(8.9)$ & $121(13.14)$ & $<0.01$ \\
Prior arrhythmia & $194(20.7)$ & $10(1.1)$ & 0.14 \\
Prior CAD & $19(1.9)$ & & \\
Prior tachycardia & & & \\
\hline
\end{tabular}

Data are number (\%) except where otherwise specified.

$\mathrm{CABG}$, coronary artery bypass graft; $\mathrm{CAD}$, coronary artery disease; CHF, congestive heart failure; MI, myocardial infarction. 
Table 2 Summary statistics on cost and length of stay for the traditional approach

\begin{tabular}{|c|c|c|c|}
\hline Risk stratification & $\begin{array}{l}\text { Number of patients } \\
n=938(\%)\end{array}$ & $\begin{array}{l}\text { Cost Median } \\
\text { (25-75th centile) }\end{array}$ & $\begin{array}{l}\text { Hours in hospital } \\
\text { Median (25-75th centile) }\end{array}$ \\
\hline Low & $9(1.0)$ & \$A1636 (\$A1155-\$A3592) & $11.5(9.5-31.5)$ \\
\hline Intermediate & $585(62.4)$ & \$A1961 (\$A1466-\$A3780) & $24.6(9.9-35.1)$ \\
\hline EST & 356 & \$A1863 (\$A1493-\$A2528) & $23.8(10.2-28.7)$ \\
\hline Negative & 312 & \$A1799 (\$A1477-2243) & $20.4(10.1-27.8)$ \\
\hline Equivocal & 26 & \$A2700 (\$A1904-4277) & $29.7(26.0-52.1)$ \\
\hline Positive & 18 & \$A7113 (\$A5419-\$A10 348) & $61.8(34.5-130.5)$ \\
\hline No EST & 229 & \$A2974 (\$A1294-\$A7163) & $27.6(8.5-76.7)$ \\
\hline Send home & 101 & \$A1285 (\$A1094-\$A1626) & $8.4(6.2-10.4)$ \\
\hline Admit to ward & 128 & \$A6642 (\$A3975-\$A9085) & $71.0(34.2-126.7)$ \\
\hline High & $336(35.8)$ & $\$ A 6743$ (\$A2755-\$A12 509) & $73.2(27.5-143.7)$ \\
\hline Alive with treatment & 331 & \$A6705 (\$A2755-\$A12 495) & $72.3(27.0-142.4)$ \\
\hline Died $<30$ days & 5 & \$A9340 (\$A3177-\$A38 594) & $146.4(83.4-426.5)$ \\
\hline Left against medical advice & $8(0.8)$ & $\$ A 1461$ (\$A1057-\$A2232) & $14.1(5.5-25.0)$ \\
\hline
\end{tabular}

hypertension, dyslipidaemia and family history of CAD. Moreover, the proportion of patients having prior medical conditions was higher among the traditional approach group.

\section{Cost and length of stay analysis}

In the traditional approach $(\mathrm{n}=938),<1 \% \quad(\mathrm{n}=9)$ were allocated to the low-risk category, 62\% (n=585) were classed as intermediate risk, $36 \%(\mathrm{n}=336)$ as high risk and $0.8 \%(\mathrm{n}=8)$ of patients left against medical advice (table 2). None of the nine low-risk patients had EST, and they spent fewer hours in hospital than intermediate-risk and high-risk patients. Among patients in the intermediate-risk group, those who had an EST incurred lower costs than those who did not (\$1863 vs $\$ 2974$ ). The difference arose as $88 \%$ of patients having an EST were discharged from hospital following a negative EST result. In contrast, $128(56 \%)$ of 229 patients who did not perform an EST were admitted to the ward for further investigation, which incurred higher costs. Five patients died, with three having a cardiovascular cause of death during their hospital stay and two dying within 6 days of hospital discharge from noncardiovascular causes.

Of the 921 patients available for the Brisbane protocol, $18 \%(\mathrm{n}=169)$ were classed as 'Brisbane protocol-low' risk, $55 \%(\mathrm{n}=514)$ as 'Brisbane protocol-intermediate' risk, $25 \% \quad(n=230)$ as high risk and $0.9 \% \quad(n=8)$ of patients left against medical advice (table 3). Overall, $50 \%$ of patients managed by the Brisbane protocol performed an EST. In comparison, $38 \%$ of the cohort in the traditional approach performed an EST. In the

Table 3 Summary statistics on cost and length of stay for the Brisbane protocol

\begin{tabular}{|c|c|c|c|}
\hline Risk stratification & $\begin{array}{l}\text { Number of patients } \\
\mathrm{n}=921(\%)\end{array}$ & $\begin{array}{l}\text { Cost Median } \\
\text { (25-75th centile) }\end{array}$ & $\begin{array}{l}\text { Hours in hospital } \\
\text { Median (25-75th centile) }\end{array}$ \\
\hline Brisbane protocol-Iow & $169(18.3)$ & \$A1061 (\$A901-\$A1374) & $5.3(4.3-7.0)$ \\
\hline EST & 39 & \$A1563 (\$A1042-\$A1807) & $7.7(6.5-24.5)$ \\
\hline Negative & 37 & \$A1515 (\$A1028-\$A1706) & 7.7 (6.4-10.4) \\
\hline Equivocal & 2 & $\$ A 3897$ & 28.9 \\
\hline No EST & 136 & $\$ A 1009$ (\$A820-\$A1233) & $4.8(4.2-5.9)$ \\
\hline Send home & 129 & \$A989 (\$A818-\$A1198) & $4.8(4.2-5.7)$ \\
\hline Admit to ward & 7 & \$A2858 (\$A1028-\$A9777) & $23.0(4.8-127.5)$ \\
\hline Brisbane protocol-intermediate & $514(55.8)$ & \$A1485 (\$A1095-\$A2086) & $7.9(6.3-15.2)$ \\
\hline EST & 420 & \$A1449 (\$A1085-\$A1759) & $7.7(6.3-10.1)$ \\
\hline Negative & 351 & \$A1366 (\$A1063-\$A1618) & $7.3(6.1-8.8)$ \\
\hline Equivocal & 47 & \$A3111 (\$A1770-\$A5492) & $26.8(9.6-34.3)$ \\
\hline Positive & 22 & \$A6056 (\$A4065-\$A6765) & $46.3(28.9-52)$ \\
\hline No EST & 94 & \$A2840 (\$A1143-\$A7838) & $27.5(6.2-53.4)$ \\
\hline Send home & 42 & \$A1116 (\$A942-\$A1436) & $621(4.7-8.5)$ \\
\hline Admit to ward & 52 & \$A6856 (\$A4178-\$A11 238) & $50.8(29.5-80.0)$ \\
\hline High & $230(25.0)$ & \$A5626 (\$A2655-\$A9545) & $43.7(24.4-74.8)$ \\
\hline Left against medical advice & $8(0.9)$ & $\$ A 1272(\$ A 1168-\$ A 1737)$ & $6.0(5.2-7.3)$ \\
\hline
\end{tabular}


'Brisbane protocol-low'-risk group, 39 of 169 patients performed an EST, while 420 of 514 in the 'Brisbane protocol-intermediate'-risk group had an EST. Patients in the 'Brisbane protocol-low'-risk group incurred fewer costs and spent fewer hours in hospital than those in the 'Brisbane protocol-intermediate'-risk group (\$1061 vs $\$ 1485 ; 5.3 \mathrm{~h}$ vs $7.9 \mathrm{~h}$ ). Patients who left against medical advice incurred the least cost. No one died within 30 days after discharge in the Brisbane protocol cohort.

In table 4, costs and hospital length of stay according to admission category were compared between the traditional approach group and the Brisbane protocol group. Nearly $83 \%$ of patients assessed by the Brisbane protocol were admitted to ED only and ED short stay unit compared with $66 \%$ in the traditional approach group. Total hospital length of stay was shorter with the Brisbane protocol. Fewer patients in the Brisbane protocol group received inpatient care $(17 \%$ vs $33 \%)$, and they had generally shorter lengths of stay, median 45 vs $52.5 \mathrm{~h}$. The median cost and length of stay when considering all patients were lower in the Brisbane protocol cohort.

\section{Percentage of patients discharged within $4 \mathrm{~h}$ from ED}

The percentage of patients who were discharged from ED within $4 \mathrm{~h}$ by risk stratification is shown in table 5 , and we give the results before and after patient characteristics in the traditional approach were adjusted in an attempt to make the two cohorts more comparable. As the Brisbane protocol only further stratified low-risk and intermediate-risk groups, the proportion of patients discharged from ED in high-risk group was similar between two approaches. Although the Brisbane protocol failed to achieve NEAT and discharged, admitted or transferred $62 \%$ of ED patients from all risk groups within $4 \mathrm{~h}$, it enabled physicians to discharge a higher proportion of patients within $4 \mathrm{~h}$ in low-risk and intermediate-risk groups than the traditional approach ( $72 \%$ vs $51 \%)$.

\section{Decision tree model outputs}

The expected costs and length of stay in hospital of the two approaches from the decision tree model are shown in table 6 . The average patient managed by the Brisbane protocol cost $\$ 1229$ less, and $26 \mathrm{~h}$ in hospital was saved compared to the traditional approach. These differences are shown by the probabilistic sensitivity analysis and are plotted in figure 4 .

Figure 5 provides the proportion of the 10000 resamples where the Brisbane protocol resulted in a lower cost or shorter stays for the average patient. When only cost was taken into consideration, the Brisbane protocol had a $78 \%$ probability of incurring fewer costs. When shorter length of stay was the decision criteria, there was a $79 \%$ probability the Brisbane protocol is optimal.

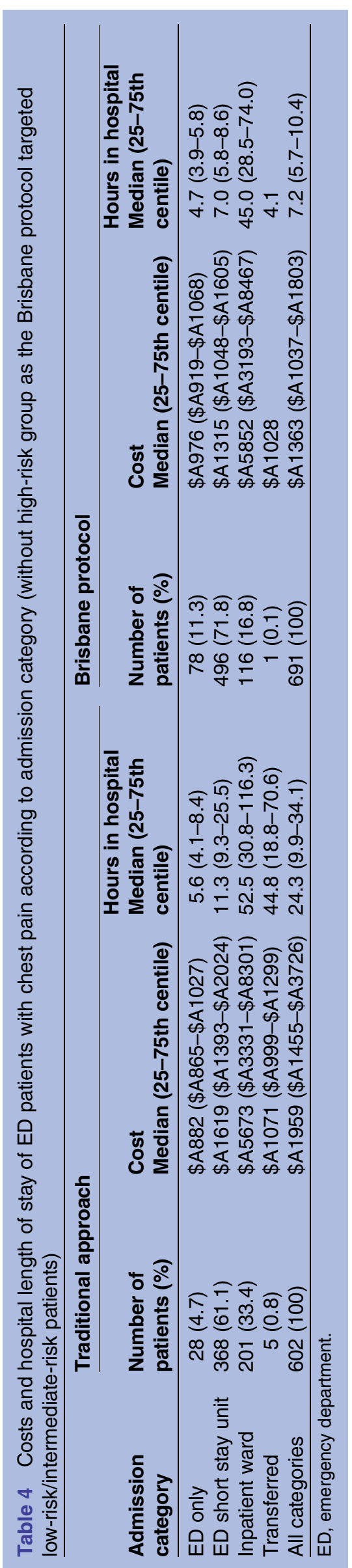


Table 5 Percentage of patients discharged from ED within $4 \mathrm{~h}$ by risk stratification before and after baseline characteristics were adjusted

\begin{tabular}{llll}
\hline & $\begin{array}{l}\text { Traditional } \\
\text { approach } \\
\text { (not } \\
\text { adjusted), \% }\end{array}$ & $\begin{array}{l}\text { Traditional } \\
\text { approach } \\
\text { (adjusted), }\end{array}$ & $\begin{array}{l}\text { Brisbane } \\
\text { protocol } \\
\text { (\%) }\end{array}$ \\
\hline High risk & 26.0 & 30.1 & 30.2 \\
Low and & 46.1 & 50.6 & 72.3 \\
intermediate risk & & & \\
\hline ED, emergency department. & &
\end{tabular}

\section{DISCUSSION}

We report the first study of the potential health services gain of adopting an ADP into routine practice in the Australian healthcare setting. Some advantages of ADP for assessing patients presenting to ED with chest pain have previously been demonstrated. ${ }^{6-8}$ This analysis used data collected over two different periods and included it in a decision tree model to compare cost and length of stay between the traditional assessment approach and the Brisbane protocol. We demonstrated the economic benefits of applying the Brisbane protocol in a hospital setting.

The Brisbane protocol for the assessment of emergency patients with possible cardiac chest pain may have considerable benefits to patients with early notification about the underlying cause of their symptoms, and early discharge of those without a cardiac diagnosis. Adopting the Brisbane protocol could also assist in meeting NEAT targets. About $70 \%$ of non-high-risk patients could be assessed rapidly for ACS and discharged from ED within $4 \mathrm{~h}$ under the Brisbane protocol. In the hospital, the average ED length of stay fell from 289 min between 2008 and 2010 to 243 min between 2011 and 2014, the period when the Brisbane protocol was implemented. Whether this observed saving of 45 min per patient was caused by the Brisbane protocol cannot be known for certain due to the non-randomised study design. The overall capacity released for the hospital was substantial, with a reduction in the expected assessment period from 42 to $16 \mathrm{~h}$ for all non-high-risk patients. The reduction in need for lengthy admission supported same-day discharge for many patients. The economics of this in terms of time missed from work, family and social activities is hard to quantify; however, early discharge home for patients is likely to have had a positive effect on patient satisfaction.

The Brisbane protocol identified a large proportion of patients as low risk. This is a significant increase by comparison with the current National Heart Foundation and Cardiac Society of Australia and New Zealand Guidelines risk stratification process and is an equivalent-sized low-risk cohort in comparison with other risk scores such as TIMI and GRACE scores when used for ED patient assessment. The true reduction in need for EST testing in this cohort may have larger system effects in terms of improving access for other patients requiring this cardiac investigation. This was not assessed in the study. Compared to other ADP approaches, the Brisbane protocol has its strength that it incorporates both AMI and UAP. There are other approaches used to identify those at risk of AMI alone ${ }^{19}{ }^{20}$ but these ignore the increased short-term to medium-term risk of recurrent ischaemic events in those with underlying CAD and UAP. Moreover, the tools required for implementation of the Brisbane protocol do not differ from what is currently widely available. Troponin assays and ECGs will continue to be performed, and the risk stratification process can be easily adopted in other hospitals. We believe that the uptake of this strategy into clinical practice will be rapid.

Other economic analysis of applying ADP to assess patients with chest pain also shows evidence for reduced hospitalisation stay and lower costs. Asher $e t a l^{21}$ in Israel examined the clinical outcomes and cost-effectiveness of an ADP using contemporary technology versus routine care and found that an ADP could save time and resources. There was a slight decrease in total costs when patients were treated ADP, but the difference was not significant. Compared with their comparative prospective study, our study has strengths in that we combined comparative study with an economic decision model. By taking account of the probability of being classified as low or intermediate risk and the probability of having an EST, the decision tree model demonstrates the expected cost and length of stay for a patient who presents to ED with chest pain. In addition, we conducted probabilistic sensitivity analysis to account for parameter uncertainty surrounding cost and length of stay. The Brisbane protocol has shown a high probability

Table 6 Expected costs and length of stay in hospital per patient for the traditional approach and the Brisbane protocol (without high-risk group as the Brisbane protocol targeted low-risk/intermediate-risk patients)

\begin{tabular}{|c|c|c|c|c|}
\hline & $\begin{array}{l}\text { Expected cost } \\
(95 \% \mathrm{Cl})\end{array}$ & $\begin{array}{l}\text { Expected length } \\
\text { of stay }(95 \% \mathrm{Cl})\end{array}$ & $\begin{array}{l}\text { Incremental } \\
\text { cost }(95 \% \mathrm{Cl})\end{array}$ & $\begin{array}{l}\text { Incremental length } \\
\text { of stay }(95 \% \mathrm{Cl})\end{array}$ \\
\hline $\begin{array}{l}\text { Traditional } \\
\text { approach }\end{array}$ & $\begin{array}{l}\text { \$A3454 (\$A1438- } \\
\text { \$A7159) }\end{array}$ & 42 h $(8-153$ h) & & \\
\hline Brisbane protocol & $\begin{array}{l}\text { \$A2225 (\$A1282- } \\
\text { \$A3609) }\end{array}$ & $16 \mathrm{~h}(7-32$ h) & $\begin{array}{l}-\$ A 1229 \\
(-\$ A 5122 \text { to } \\
\$ A 1266)\end{array}$ & $\begin{array}{l}-26 \mathrm{~h}(-136 \text { to } \\
14 \mathrm{~h})\end{array}$ \\
\hline
\end{tabular}



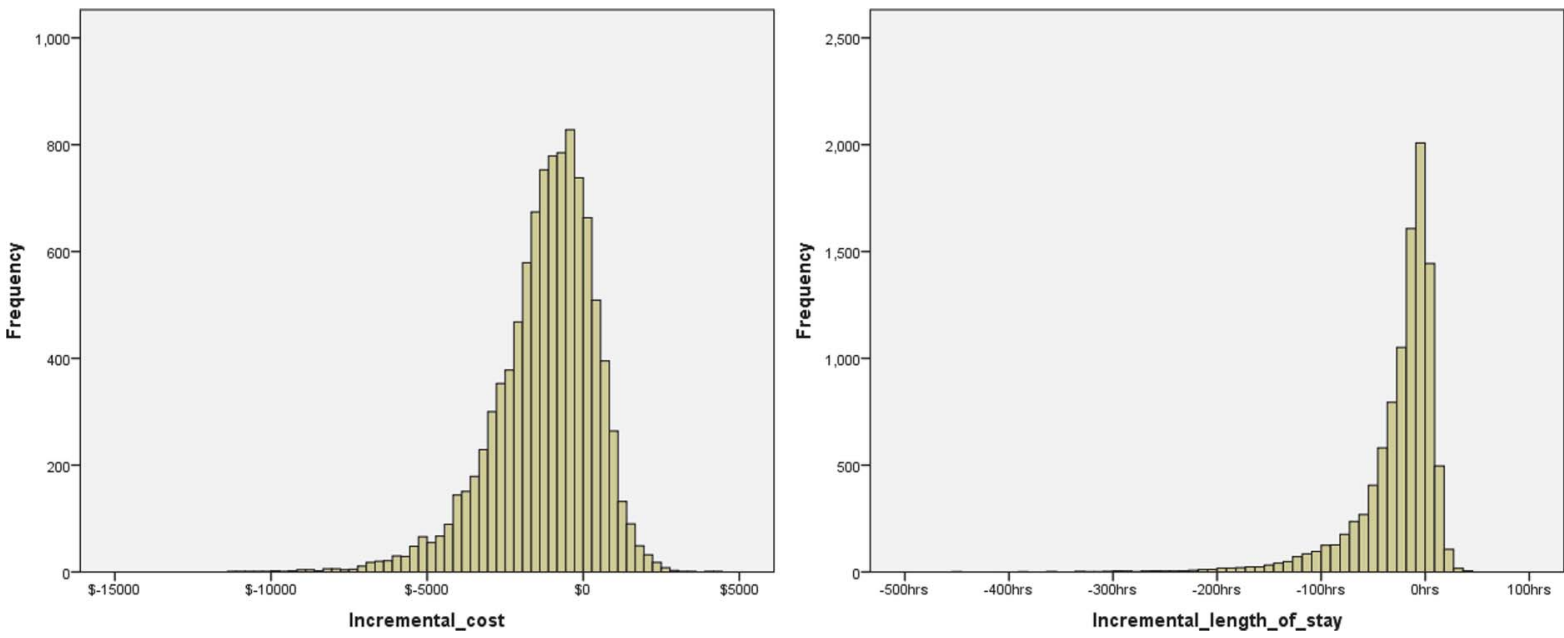

Figure 4 Distributions of incremental cost $(\$ A)$ and length of stay for the Brisbane protocol with the traditional approach as the reference from the 10000 probabilistic sensitivity analyses.
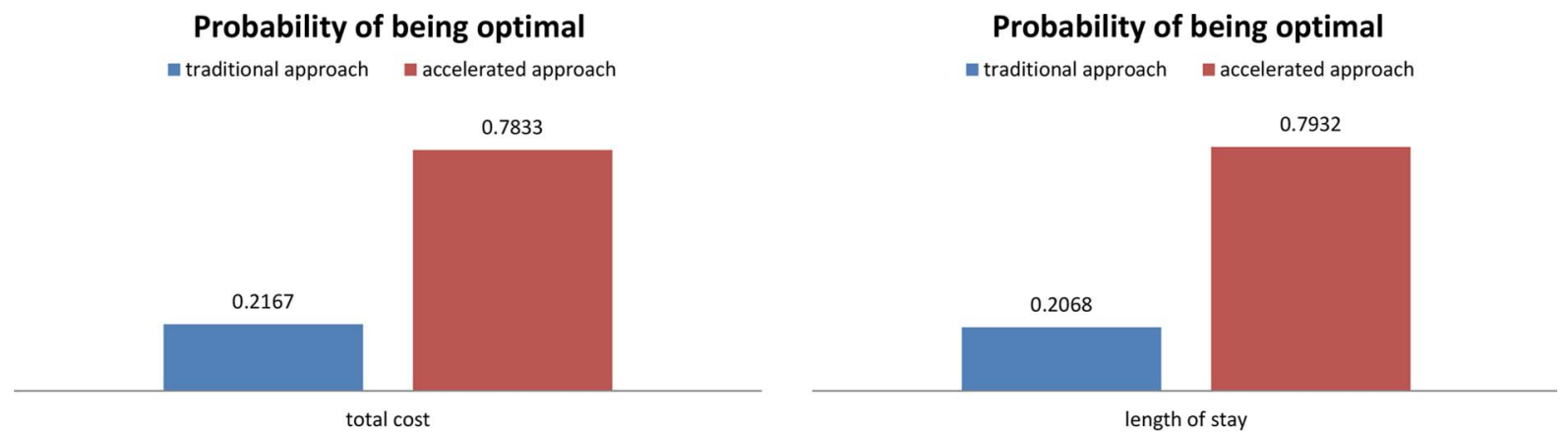

Figure 5 Probability of an approach being optimal in terms of cost and length of stay from the 10000 probabilistic sensitivity analyses.

of being optimal compared with the traditional approach.

The limitations of this analysis should be acknowledged. First, in both cohorts, patients were recruited between 8:00 and 17:00 due to the significant cost of out-of-hour recruitment. The potential impact of enrolling patients for a portion of the day is not known as we are unable to quantify any possible effect without data from out-of-hour patients. However, we do not believe the impact of predominantly in-hour recruitment will have a significant impact on the findings. One of our previous studies examined whether in-hour recruitment biased the findings. ${ }^{22}$ We found that individuals recruited outside work hours did not differ from those recruited within work hours in terms of demographics and medical history. Second, ideally a pragmatic parallel multicentre randomised controlled trial would be done, but this would cost millions of dollars and will take time to organise. With the observational design, we cannot be sure that the Brisbane protocol contributed to the differences in the outcomes. The results of the adjustment (table 5) provide some evidence of an effect arising from the Brisbane protocol. When the two cohorts were adjusted for the baseline variables, the proportion of patients discharged from ED within $4 \mathrm{~h}$ did change, but not dramatically. Despite these limitations, the improvement in cost and length of stay outcomes is plausible, and the purpose of this study is to provide data that contribute to a decision being made, rather than perfectly estimating the size of an effect. As this study is focused on the health economic outcomes of the Brisbane protocol, it does not report the detailed clinical outcomes of patients managed according to the traditional diagnostic approach and the Brisbane protocol.

\section{CONCLUSION}

The Brisbane protocol may be a cost-saving change to services for the assessment of ED patients with possible ACS. Patients and the EDs that manage them might benefit from this system of care.

\section{Author affiliations}

${ }^{1}$ Institute of Health and Biomedical Innovation, Queensland University of Technology, Brisbane, Queensland, Australia

${ }^{2}$ Royal Brisbane and Women's Hospital, Brisbane, Queensland, Australia

${ }^{3}$ The University of Queensland, Brisbane, Queensland, Australia

${ }^{4}$ Department of Emergency Medicine, Baylor College of Medicine, Houston, Texas, USA 
Twitter Follow Adrian Barnett at @aidybarnett

Contributors LC, JHG, WAP, WFP, NG, AGB and KM led the design of the study. Data analysis was undertaken by QC, JHG, AGB and KM. All authors critically reviewed each draft of the manuscript. The final version was approved by all authors.

Funding This study was funded by the Queensland Emergency Medicine Research Foundation (QEMRF). LC was supported by a fellowship from QEMRF.

\section{Competing interests None declared.}

Provenance and peer review Not commissioned; externally peer reviewed.

Data sharing statement No additional data are available.

Open Access This is an Open Access article distributed in accordance with the Creative Commons Attribution Non Commercial (CC BY-NC 4.0) license, which permits others to distribute, remix, adapt, build upon this work noncommercially, and license their derivative works on different terms, provided the original work is properly cited and the use is non-commercial. See: http:// creativecommons.org/licenses/by-nc/4.0/

\section{REFERENCES}

1. Centers for Disease Control and Prevention. National Hospital Ambulatory Medical Care Survey: 2011 Emergency Department Summary Tables. 2011. http://www.cdc.gov/nchs/data/ahcd/ nhamcs emergency/2011 ed web tables.pdf

2. Bhuiya FA, Pitts SR, McCaig LF. Emergency department visits for chest pain and abdominal pain: United States, 1999-2008. NCHS Data Brief 2010;43:1-8.

3. Acute Coronary Syndrome Guidelines Working Group. National Heart Foundation of Australia and Cardiac Society of Australia and New Zealand Guidelines for the Management of Acute Coronary Syndromes. Med J Aust 2006;184:1-32.

4. Cullen L, Greenslade J, Merollini K, et al. Cost and outcomes of assessing patients with chest pain in an Australian emergency department. Med J Aust 2015;202:427-32.

5. Council of Australian Governments. National Health Reform Agreement - National Partnership Agreement on Improving Public Hospital Services. 2011. http://www.federalfinancialrelations.gov.au/ content/npa/health_reform/national-workforce-reform/national partnership.pdf

6. Than M, Cullen L, Reid CM, et al. A 2-h diagnostic protocol to assess patients with chest pain symptoms in the Asia-Pacific region (ASPECT): a prospective observational validation study. Lancet 2011;377:1077-84

7. Than M, Cullen L, Aldous S, et al. 2-Hour accelerated diagnostic protocol to assess patients with chest pain symptoms using contemporary troponins as the only biomarker: the ADAPT trial. J Am Coll Cardiol 2012;59:2091-8.

8. Cullen L, Mueller C, Parsonage WA, et al. Validation of high-sensitivity troponin I in a 2-hour diagnostic strategy to assess 30-day outcomes in emergency department patients with possible acute coronary syndrome. J Am Coll Cardiol 2013;62:1242-9.

9. George T, Ashover S, Cullen L, et al. Introduction of an accelerated diagnostic protocol in the assessment of emergency department patients with possible acute coronary syndrome: the Nambour Short Low-Intermediate Chest pain project. Emerg Med Australas 2013;25:340-4.

10. Than M, Aldous S, Lord SJ, et al. A 2-hour diagnostic protocol for possible cardiac chest pain in the emergency department: a randomized clinical trial. JAMA Intern Med 2014;174:51-8.

11. Aldous SJ, Richards MA, Cullen L, et al. A new improved accelerated diagnostic protocol safely identifies low-risk patients with chest pain in the emergency department. Acad Emerg Med 2012;19:510-16.

12. Queensland Health. The Accelerated Chest Pain Risk Evaluation (ACRE) Project. Secondary the Accelerated Chest Pain Risk Evaluation (ACRE) Project. https://www.health.qld.gov.au/caru/html/ acre.asp

13. Luepker RV, Apple FS, Christenson RH, et al. Case Definitions for Acute Coronary Heart Disease in Epidemiology and Clinical Research Studies: a Statement From the AHA Council on Epidemiology and Prevention; AHA Statistics Committee; World Heart Federation Council on Epidemiology and Prevention; the European Society of Cardiology Working Group on Epidemiology and Prevention; Centers for Disease Control and Prevention; and The National Heart, Lung, and Blood Institute. Circulation 2003;108:2543-9.

14. Cullen L, Than M, Brown AF, et al. Comprehensive standardized data definitions for acute coronary syndrome research in emergency departments in Australasia. Emerg Med Australas 2010;22:35-55.

15. Greenslade JH, Cullen L, Kalinowski L, et al. Examining renal impairment as a risk factor for acute coronary syndrome: a prospective observational study. Ann Emerg Med 2013;62:38-46.e1.

16. Lumley T. Analysis of complex survey samples. J Stat Softw 2004;9:1-19.

17. Dodd S, Bassi A, Bodger K, et al. A comparison of multivariable regression models to analyse cost data. J Eval Clin Pract 2006;12:76-86.

18. AlHW. Australia's health 2014. Canberra: AlHW, Australia's health series no. 14. Cat. no. AUS 178, 2014.

19. Body R, Carley S, McDowell G, et al. The Manchester Acute Coronary Syndromes (MACS) decision rule for suspected cardiac chest pain: derivation and external validation. Heart 2014;100:1462-8.

20. Reichlin T, Cullen L, Parsonage WA, et al. Two-hour algorithm for triage toward rule-out and rule-in of acute myocardial infarction using high-sensitivity cardiac troponin T. Am J Med 2015;128:369-79.e4.

21. Asher $\mathrm{E}$, Reuveni $\mathrm{H}$, Shlomo $\mathrm{N}$, et al. Clinical outcomes and cost effectiveness of accelerated diagnostic protocol in a chest pain center compared with routine care of patients with chest pain. PLOS ONE 2015;10:e0117287.

22. Cullen L, Parsonage WA, Greenslade J, et al. Comparison of early biomarker strategies with the Heart Foundation of Australia/Cardiac Society of Australia and New Zealand guidelines for risk stratification of emergency department patients with chest pain. Emerg Med Australas 2012;24:595-603. 